\title{
Improvement of responsiveness at a clinic setting in secondary care Hospital in Sri Lanka
}

\author{
Ranga Sabhapathige*, D. A. R. K. Dilrukshi ${ }^{* *}$ \\ ${ }^{*}$ CHSS, University of Kent \\ ** CHSS, University of Kent \\ DOI: 10.29322/IJSRP.11.07.2021.p11562 \\ http://dx.doi.org/10.29322/IJSRP.11.07.2021.p11562
}

\begin{abstract}
Introduction

Responsiveness is defined as "how well the health system meets the legitimate expectations of people for non-health aspect of health care. Improving the training of health care workers in health system responsiveness, improve the processes at health setting and providing basic amenities are very important to achieve high level of responsiveness.
\end{abstract}

\section{Aims}

To improve the level of responsiveness at the medical clinic in base hospital, Balapitiya and to develop and implement strategies to improve the level of responsiveness

\section{Methodology}

This is interventional research project conducted from December 2018 to June 2019 in medical clinic at base hospital Balapitiya. Patients who were treated more than 3 months at MC in BHB were included in this project. Data collected by using interviewer administered structured questionnaire, KII and structured format to assess the waiting time in both pre and post-tests.

Study conducted in three components including pre-interventional assessment, intervention and assessment of post-interventional improvement of health system responsiveness. Post-test sample was compared with pre-test sample by using independent t-test.

\section{Results}

The overall responsiveness had significantly improved in the post-test sample $(p=0.001)$. The specific domains like dignity $(p=0.00)$, communication $(\mathrm{p}=0.00)$, confidentiality $(\mathrm{p}=0.00)$ and prompt attention $(\mathrm{p}=0.00)$ improved significantly. Although, three domains including autonomy $(\mathrm{p}=0.130)$, choice of provider $(\mathrm{p}=0.786)$ and basic amenities $(\mathrm{p}=0.174)$ were not improved significantly.

\section{Conclusions}

Findings of this project indicate that improving the knowledge, attitudes of health care workers through comprehensive training programs in health system responsiveness improving the processes at health care settings and providing basic amenities to the patients will improve responsiveness. It further recommended adopting the intervention in other units of the hospital to improve overall health system responsiveness.

Key words: Responsiveness, Medical clinic, Process improvement,

\section{INTRODUCTION}

$\mathrm{H}$ ealth system Responsiveness (HSR) is defined as how good the health system complies with the legitimate expectations of the customers for the non-health enhancing aspects of health care. It contains eight elements including dignity, confidentiality, autonomy, prompt attention, social support, basic amenities, choice of provider and communication. The social support element was considered relevant to inpatient care settings only (1).

Patients expect that health care institutes must treat them with dignity, should support them in making decisions regarding their treatment, good communication with health care staff and assure that their medical records are kept confidential, in addition to making them healthy. They also feel that health care staff must provide prompt attention, access to social support, choice of provider and basic amenities of adequate quality. The improvement in responsiveness of health system does not need big investment, sophisticated technology or change in legislation. A small training programme to change the attitudes of HCWs to respect the patient's dignity and autonomy, can improve the responsiveness of the health system to greater extent (1).

A study conducted in Teheran revealed that hospital responsiveness had a strong effect on overall patient satisfaction. Quality of basic amenities and respect for human dignity were the most powerful domains influencing overall patient satisfaction. In developed countries, 
researches explain that satisfied customers are more likely to comply with treatments and they will also provide reliable information to health care provider and continue to use medical services (2). There is evidence also from developing countries that patient satisfaction will influence the utilization of health services and compliance with doctors' recommendations (3).

The report on "Changing Mindsets - Strategy on Health Policy and Systems Research" of the World health organization pointed out that responsiveness is a well-recognized key objective of most of the health systems at present (4).

The most commonly used framework for measuring HSR was developed by the WHO (1). It contains seven elements against which responsiveness is measured. Those are dignity, autonomy, confidentiality, prompt attention, quality of amenities, access to social support networks and choice of service provider.

HSR varies among countries with different socio-economic conditions (5). WHO survey on HSR conducted in 191 countries revealed that countries spending higher amounts of money on health have high level of HSR when compared to the countries which spent less. Sri Lanka was ranked in the $95^{\text {th }}$ place with a responsiveness index of 5.08 in this study. Health system of United States of America rank as best with 8.10 in responsive indexes according to the results of this study, (5).

$\mathrm{MoH}$ introduced health care quality and safety concepts to Sri Lankan health system during last two decades. Responsiveness was included as one dimension of quality, but still there was no priority for it. However, National health policy document identified responsiveness as a one of major challenges faced by Sri Lankan health system (6).

Few studies have been conducted in Sri Lanka on Health system responsiveness. Sanjeewani (7) indicated that there is a potential to obtain excellent improvement in level of responsiveness in government health institutions of Sri Lanka, regardless of limited resources, if due attention was paid by service provider. Somarathna (8) conducted an interventional research project to improve responsiveness of nursing officers in inward setup at DGH Gampola. In that project, he found that level of responsiveness among nursing officers could be increased through properly planned training module. Ranasinghe (9) conducted a study at medical wards of DGH, Matara to assess the HSR. Perera et al.(10) studied the level of HSR at 38 family planning clinics in Colombo district. There is no research project conducted in Sri Lanka to improve the level of HSR at a hospital clinic setup. Therefore, this research project would be the first project conducted in country to assess the level of responsiveness at a hospital clinic set up and to plan and implement an intervention to improve the HSR.

Survey conducted in 35 countries by De Silva and Valentine (5) had identified that the average overall responsiveness score for Sri Lanka as 4.82 (scale 0 to 10) and the scores for all domains of responsiveness were also low. Dignity, Autonomy and confidentiality scores were 4.31, 3.73, and 5.26 (scale 1 to 10 ) respectively. Domains of prompt attention and quality of basic amnesties obtained 4.80 and 3.41 (5). These figures indicated that there is still lot of room for improvement of responsiveness in Sri Lanka.

Base Hospital, Balapitiya (BHB) is the largest base hospital of this type in the southern province and has 440 patient beds. It caters around 300,000 population in Galle district. Hospital management has taken several initiatives to improve health care quality of the hospital during last few years. Although, responsiveness to the patient's expectations has still not improved to the standards according to anecdotal sources, especially at the medical clinic (MC). Improving the knowledge, attitudes and practices of HCW in HSR, improving the processes at health settings and providing basic amenities to the patients are very important to achieve high level of HSR (11). Thus, this interventional research project is designed to improve knowledge, attitudes and practices of HCW on HSR, improve the processes and providing basic amenities to the patients at MC in BHB.

\section{OBJECTIVES}

1. To assess the current level of responsiveness at the medical clinic in base hospital, Balapitiya.

2. To develop and implement strategies taking into consideration, respect for human rights and client orientation' to improve responsiveness at medical clinic in base hospital, Balapitiya.

3. To assess the level of improvement in responsiveness after interventions at the medical clinic in base hospital, Balapitiya.

\section{$\underline{\text { 2.1 Project Design }}$}

\section{METHODOLOGY}

Interventional research project designed to improve the level of HSR at MC in BH, Balapitiya. The project contained three components. Component 01: The current level of responsiveness at MC of BHB was assessed by using, Interviewer administered structured questionnaire to assess the patient perception, KII with selected hospital staff members (Medical superintendent, VP, Matrons, Nursing in charge, Medical officers and overseer), process mapping of medical clinics to identify the delays and non-value added steps in medical clinic, patient waiting time calculation

Component 02: Designed and implemented an intervention to improve the level of responsiveness at MC of BHB.

Component 03: Post interventional condition, pre and post intervention comparison to see the improvement was calculated by using, the same structured questionnaire, KII with above team, waiting time calculation

\subsection{Project setting}

Medical clinic of base hospital, Balapitiya

\subsection{Project period}

Project period was 01 st of December 2018 to $15^{\text {th }}$ of June 2019. 


\subsection{Project population}

MC patients who were treated more than 3 months at MC in BHB were included in this project. KII was held with relevant staff members $(\mathrm{n}=12)$.

\subsection{Calculation of sample size and Sampling technique}

Sample size (n) was calculated by using Lwanga and Lemeshow (1991) formula given below (population prevalence 0.5, precision 0.05, and confidence level 95\%). Required sample size was 384. Purposive sampling technique was used to find the members for KII ( $\mathrm{n}=12$ ). A simple random sample of 85 patients were selected for WT assessment.

\subsection{Project instruments}

1. Interviewer administered structured questionnaire

Interviewer administered structured questionnaire was used to assess the current level of responsiveness according to the perception of patients at MC. Expert opinions and analytical guidelines issued by WHO in 2003 to assess the health system responsiveness in multi country survey study and several previously developed questionnaires on responsiveness were used (adopted) in developing the present questionnaire.

2. KII with above mentioned sample of staff members. $(n=12)$

3. Structured format was used to calculate the time taken for each step from arrival at clinic premises to leaving the pharmacy after taking the drugs.

2.7 Mode of conducting the project.

2.7.1 Pre intervention (Component 1): Patients were interviewed by using interviewer administered structured questionnaire to assess the current level of responsiveness. KII with previously mention sample was conducted. Process mapping of medical clinics was conducted to identify the delays and non-value added steps in medical clinic

\section{Process map of medical clinic at BHB}

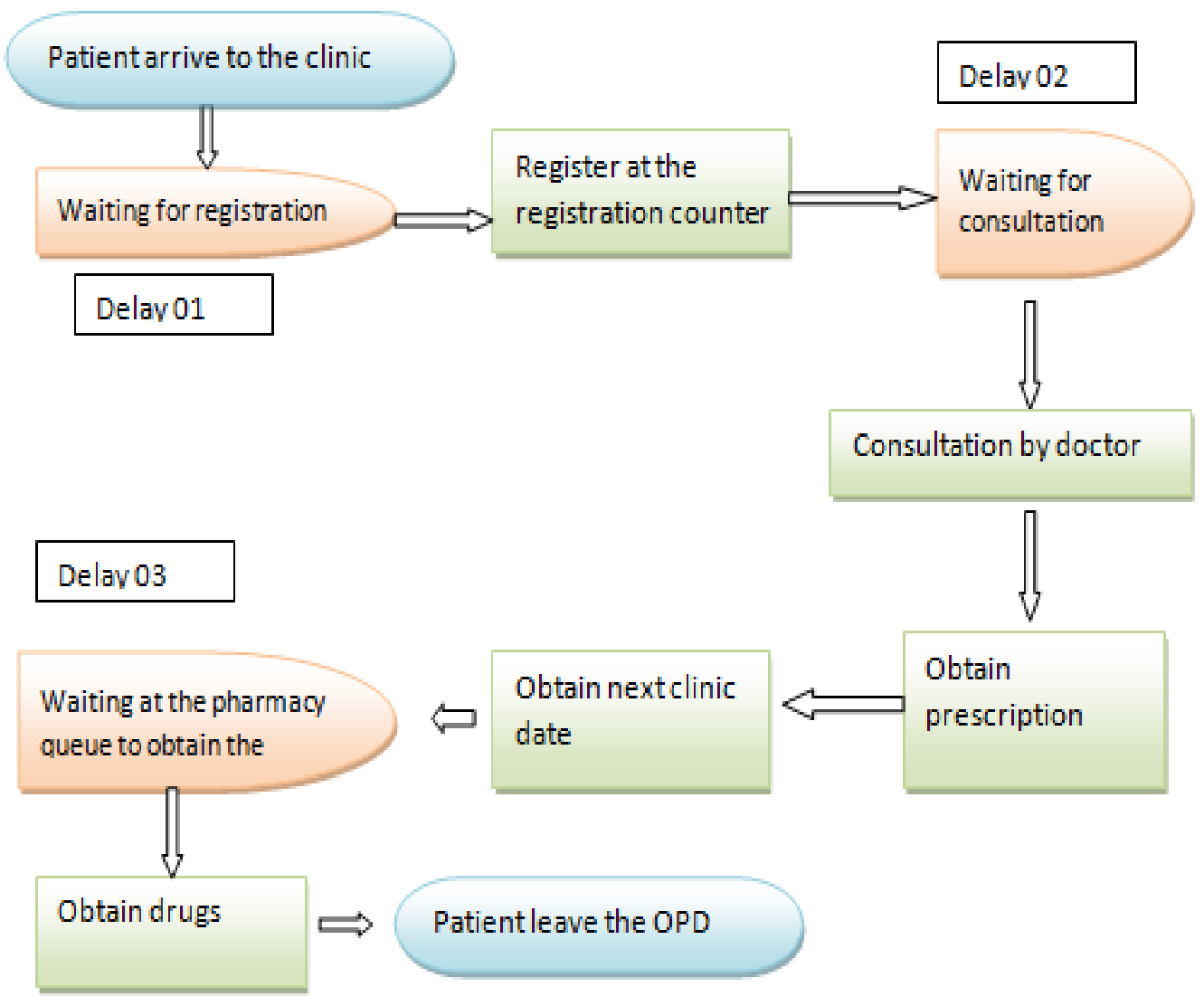

During this process mapping, three main delays were identified. Those delays and causes for them are explained in Annexure 01 Current patient waiting time was assessed by using the structured format. 


\subsubsection{Intervention (Component 2)}

a) Training programmes were conducted to improve the responsiveness of the staff. There were four separate training programs on HSR, doctor patient relationship, assertive communication, positive thinking and motivation.

Lectures, TV documentaries, video clips, role plays and discussions were included in those training sessions. Duration of the training session was 5 hours.

b) Process, Lay out and basic amnesties of MC were improved according to the findings of process mapping and KII to enhance the responsiveness. These are mentioned below; 
Process improvement

- Appointment system was streamlined. Date, time block and number were
given to the patients.

3

- Two registration desks and two HCA were placed to register the patients.

- IHOs and SHOs of both medical units (8) were placed at clinic days to see the patients.

- Three digital pressure meters were introduced and measure the blood pressure of the patients by nursing officers, before the patients enter into the consultation room.

- New exit door was built at clinic room to maintain the unidirectional flow of the patients in consultation room.

- Third pharmacy counter was opened by arranging the pharmacist from nearby hospital and counters were kept open since 8.30 a.m.

- Seating facilities of the waiting area 1 and 2 was increased by providing more benches.

9

- Water filter with drinking water was provided to medical clinic.

- Broken toilets were repaired and improve the cleanliness of toilets by better supervision.

- Unwanted condemning items were removed from the clinic. Clinic was rearranged to encourage the unidirectional flow of patient ant to minimize the congestion.

- Outlook of the clinic was improved by painting and cleaning the surroundings.

2.7.1 Post intervention (Component 3): Post-test responsiveness was assessed by using the same questionnaire used in pre intervention stage, KII with same team and Improvement in waiting time after intervention was assessed by using same format.

2.8 Methods to improve Validity and reliability of Data

Face validity and content validity of the questionnaire were assessed by the experts in the subjects. Pre-testing of the questionnaire conducted to enhance the validity of the instrument. Test-Retest was conducted to see the reliability of the instrument. 


\subsection{Data Analysis}

SPSS 21, statistical software was used to analyze the quantitative data. Statistical significance of pre and post interventional difference was tested by using independent t-test. Qualitative data was analyzed after coding them and grouping them into common areas.

\subsection{Ethical consideration}

Ethical clearance for the project was obtained from ethical review committee of Faculty of Medicine, University of Colombo.

\section{RESULTS}

This interventional research project was conducted at the BHB from 02.01.2019 to 15.06.2019. The main objective of this project was to improve the current level of responsiveness at the medical clinic of $\mathrm{BH}$, Balapitiya. This project includes both qualitative as well as the quantitative components

\subsection{Background characteristics of the participants in pre and post samples}

As shown in table 01, majority of the participants were aged 61-70years and majority were female. When considering the educational level, majority are in grade 1-5 group. Most of the participants had received less than 5000 rupees as their monthly income. Patients with Diabetes Mellitus, Hypertension and IHD were the most prevalent disease groups.

There were no statistically significant difference observed in the distribution of age, sex, education level and monthly income of patients in pre and post samples $(P=0.662,0.958,0.561,0.570$ respectively). Therefore, the two samples can be considered as similar to each other. Background characteristics of participants in both pre and post- test are given in table 01 .

\subsection{Pre interventional stage}

\section{A. Results of the qualitative components of the Project}

Key informants' interviews (KII) to assess current level of responsiveness at the Medical clinic of BH, Balapitiya.

Twelve members participated for KII $(n=12)$. Eight out of twelve participants stated that the level of responsiveness is bad, while two stated that it is satisfactory and other two stated that responsiveness was good at medical clinic in BHB. All the participants think that staffs at medical clinic don't have enough knowledge and training on responsiveness. Except one participant, all others felt that the attitudes of the clinic staff were bad.

All participants $(n=12)$ felt that they don't have enough facilities to maintain a good level of responsiveness. They said that they don't have enough examination rooms, screens and examining areas to secure the privacy and confidentiality of the patients. Two participants stated that seating facilities were not enough at waiting areas. They also mentioned that patients were not provided drinking water at medical clinic. Eight out of twelve participants pointed out that the cleanliness and number of toilets for the patients was not enough. Most of the participants (10/12) said that lay out of the clinic was not arranged properly and it looks very congested and untidy. They stated that because of this disorganized arrangement patients had to wait long time in the clinic and doctors faced difficulty while examining the patients. Few participants (3/12) felt that external environment was also untidy and some rooms of the clinic are filled with unwanted old items. All the key informants pointed out that external and internal environment of the clinic should be improved by removing unwanted items from the clinic premises, doing necessary repairs and arranging clinic in a systematic way.

\section{B. Results of the quantitative component}

Pre-interventional overall mean responsiveness score at medical clinic was 23.56 (13.46) and it lies within the range from 9 - 44 (Table 02).

When considering the domains of responsiveness, mean dignity score was 4.72 (scale 2-8). Mean communication score was 6.72 (scale 3-12) and autonomy score was 4.55 (scale 2-8). Mean scores for confidentiality, prompt attention and choice of provider were 4.36 (scale 2-8), 0.92 (scale 0-4), 2.29 (scale 0-4) respectively. The mean score for basic amenities was 11.61 (scale 6-24) which was not used to calculate the overall responsiveness score. Those results are given in table 03 .

\subsection{Post-interventional stage}

\section{A. Results of the qualitative components of the Project}

Key informants' interviews (KII) to assess post interventional level of responsiveness at the Medical clinic of BH, Balapitiya. The same sample used for KII in Pre interventional stage was also used in this stage.

All twelve participants $(n=12)$ stated that the level of responsiveness is improved to a good level in medical clinic after intervention. All the participants said that knowledge on responsiveness of staffs at medical clinic has improved. Except two participants, all others felt that the attitudes of the clinic staff are satisfactory now. The entire participants mentioned that staffs were trained well at intervention. Still, most participants (10/12) felt that they don't have enough facilities like enough examination rooms, screens and examining areas to secure the privacy and confidentiality of the patients to maintain the good level responsiveness.

All stated that seating facilities were improved at waiting areas. They also mention that it is good that patients were provided with drinking water at medical clinic. Most participants are now happy about the cleanliness and number of toilets for the patients.

Most of the participants (9/12) said that lay out of the clinic was improved and it looks nice and tidy. They stated that after establishing the unidirectional flow and new arrangement within clinic, waiting time was reduced drastically and patient faced less difficulty. Few participants felt that external environment is still untidy and but internal environment was improved.

All $(n=12)$ felt that their staffs had good training on health system responsiveness, communication, doctor patient relationship and positive thinking. 


\section{B. Results of the quantitative component}

Overall mean responsiveness score in post-test sample was 32.05 with range from 14- 44 (Table 04) three months after intervention. In post-test sample, score for dignity domain have increased to 6.98. Mean scores for all other domains also increased in post-test sample. Post interventional mean scores for seven domains are shown in table 05.

\subsection{Impact of intervention on post- test sample}

The impact of intervention on responsiveness at medical clinic was assessed by comparing pre and post mean score for separate domains and for overall responsiveness level by using independent $t$-test. Overall responsiveness at medical clinic was improved significantly ( $p$ $=0.001$ ) in post-test sample (table 06).

When considering the specific domains, dignity $(\mathrm{p}<0.05)$, communication $(\mathrm{p}<0.05)$, confidentiality $(\mathrm{p}<0.05)$ and prompt attention $(\mathrm{p}<$ $(0.05)$ were significantly improved after intervention.

But, three domains including Autonomy $(\mathrm{p}=0.130)$, choice of provider $(\mathrm{p}=0.786)$ and basic amenities $(\mathrm{p}=0.174)$ were not improved significantly. Impact of intervention on post-test sample for specific domains of responsiveness is shown in table 07 .

\section{Impact of intervention on patient waiting time}

Total waiting time at medical clinic at BHB was significantly reduced from 140 minutes to 86.66 minutes $(p=0.00)$. Impact of intervention on patient waiting time in each step is shown table 08.

\section{DISCUSSION}

This research project was designed to improve the level of responsiveness at the Medical Clinic of Base hospital Balapitiya. The project includes assessment of pre-interventional level of responsiveness at medical clinic, process mapping of medical clinic, designing and implementing training programme to staff, process improvement through multimodal intervention and post-test evaluation to assess the improvement.

According to KII findings, the level of responsiveness was low in pre- interventional state and it has increased considerably after intervention. They also mentioned that knowledge, training and attitudes of the staff on HSR were also improved after intervention. But they felt that their facilities are not enough to maintain the privacy of the patients even after the intervention. Basic amenities also improved to acceptable level after intervention. They also indicated that changing clinic arrangement and one way patient flow system dramatically reduced the patient delays.

When considering the pre-test sample, overall responsiveness score (23.56) was low. Except confidentiality, scores for all other domains were also low in pre-test sample. These findings were compatible with the results of study conducted by Silva and Valentine (5) in Sri Lanka. A similar project was conducted in ward settings at DGH Gampola found that the mean overall nursing care responsive score of pretest sample was relatively high (11).The current project was carried out in clinic setting and considers the responsiveness of all categories of staff, would be the reason for this difference. Study conducted in Ethiopia also found that the Hospitals performed low on domains like autonomy, choice of provider, prompt attention and basic amenities (12). According to study conducted in ward setting at Egypt, mean overall responsive score was good and confidentiality was the domain again obtained the highest value (13).

In post-interventional sample, mean overall responsiveness score had increased to 32.05. Scores for all other individual domains were also increased. Mean scores for the dignity (6.89), communication (9.64), Confidentiality (6.87) and prompt attention (1.55) showed considerable increase while scores for Autonomy (4.78), choice of provider (2.32) and basic amenities (12.14) indicated little increase. These findings were similar to the project conducted by Somarathna (9) at DGH Gampola that reports.

When compared with pre-test and post-test samples, there is significant increase in mean overall responsiveness score at post-test sample $(\mathrm{p}=0.001)$. It shows that the training programme and multimodal intervention had positive impact on mean overall responsiveness score. According to study findings, domains like dignity $(\mathrm{p}=0.000)$, communication $(\mathrm{p}=0.000)$, confidentiality $(\mathrm{p}=0.000)$ and prompt attention ( $\mathrm{p}=0.000)$ showed significant difference between the pre and post-tests.

However, domains like autonomy; choice of provider and basic amenities doesn't show any significant difference. Usually patients of Sri Lanka think that doctors know better than them and they expect doctors to take decision for their care. That might be a reason for unchanged mean autonomy score. It was very difficult to give the choice to patients to decide the doctor at crowded MC at BHB even after the intervention. That would be the reason for low mean score for choice of provider domain even after the intervention. Although some basic amenities were improved during the intervention, it was very difficult to do dramatic change due to scarcity of resources. It was the reason for low mean sore for the basic amenities domain.

Prompt attention is one of the most important domains of responsiveness. WT of MC was measured at this study to assess the prompt attention. There is significant improvement in waiting time at post interventional sample in this study $(\mathrm{p}=0.00)$. It indicated that there was a positive impact on waiting time at MC by intervention.

\section{CONCLUSIONS}

This research project was carried out to improve the Responsiveness in MC at BHB. Overall level of responsiveness and responsiveness scores for specific domains were low at MC before the intervention. Staff was not trained on responsiveness and their knowledge and attitudes were poor. Patients had to wait long at MC to get the treatments.

Following intervention, overall responsiveness and mean responsive scores for specific domains of responsiveness had been increased. When consider the specific domains of responsiveness, Dignity, communication, confidentiality and prompt attention were improved significantly after the intervention. Waiting time was also reduced significantly at MC as a result of intervention.

Two major components had been identified from this project to improve the responsiveness. 
a) Training HCW on knowledge on responsiveness, communication, doctor patient relationship, assertive communication and positive thinking

b) Multimodal intervention to improve the processes and layout of clinic

\section{References}

1. De Silva, A. (2000) ‘a framework for measuring responsiveness', WHO GPE Discussion Paper Series, (32).

2. Ahmadi Kashkoli S1, Zarei E1, Daneshkohan A1, K. S. (no date) 'Hospital responsiveness and its effect on overall patient satisfaction.', Int J Health Care Qual Assur., 9;(30(8)), p. :728-736. doi: doi: 10.1108/IJHCQA-07-2016-0098.

3. Aharony, L., \& Strasser, S. (1993). Patient satisfaction: What we know about and what we still need to explore. Medical Care Review, 50(1), 49-79.http://dx.doi.org/10.1177/002570879305000104

4. Yip W. (2015) 'MindsetS Changing', Alliance for Health Policy and Systems Research, 1(1), p. 512. doi: $10.1080 / 23288604.2015 .1091538$.

5. De Silva, A and Valentine, N(2000).Measuring responsiveness: results of key informant's survey in 35 countries. GPE Discussion Paper series No 21. Geneva: World Health Organization.

6. Government of Sri Lanka. (2010). "Mahinda chinthana" Vision for future. [online] Available at : http://www.asiantribune.com/sites/asiantribune.com/files/Mahinda_Chinthana.pdf. (Accessed on 20th August 2018)

7. Sanjeewanee, R (2016) Level of Responsiveness in outpatient clinics and identify factors affecting the perceived level of Responsiveness by patients attending clinics at Dambadeniya Base Hospital. MSc Dissertation. Postgraduate institute of Medicine, University of Colombo.

8. Somarathna,J (2017), Implementation and evaluation of a training module on improving health system responsiveness for nursing officers. MD research project,Postgraduate institute of Medicine, University of Colombo.

9. Ranasinghe JP (2012) Responsiveness in the medical wards of General Hospital Matara. MSc Medical Administration Dissertation.

10. Perera WLP. (2017) 'Health systems responsiveness and its correlates: evidence from family planning service provision in Sri Lanka', WHO South-East Asia Journal of Public Health, 1(4), p. 457. doi: 10.4103/2224-3151.207048.

11. Darby C.(2000) 'Strategy on measuring responsiveness', Health (San Francisco), (23), pp. 1-23. doi: 10.1002/ejoc.201200111.

12. Yakob B. and Ncama BP. (2017) 'Measuring health system responsiveness at facility level in Ethiopia: Performance, correlates and implications', BMC Health Services Research. BMC Health Services Research, 17(1), pp. 1-12. doi: 10.1186/s12913-0172224-1.

13. Ebrahimipour H. (2013) 'Health System Responsiveness : A Case Study of General Hospitals in egypt', 1(1), pp. 85-90. doi: 10.15171/ijhpm.2013.13. 
Table 01: Background characteristics of the participants following pre and post- tests

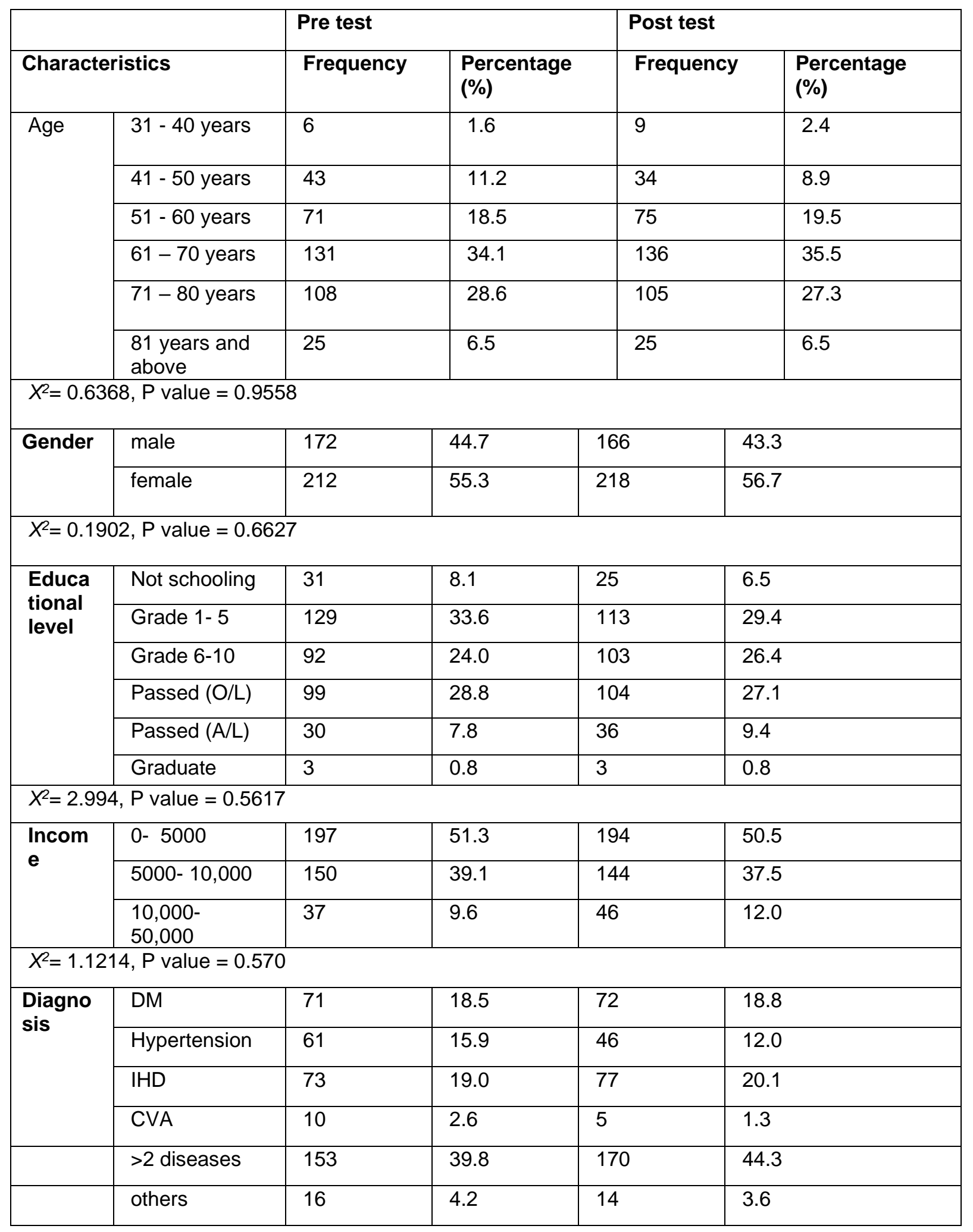


Table 02: Pre- interventional level of overall Responsiveness at medical clinic according to perception of patients

\begin{tabular}{|l|l|l|}
\hline \multirow{2}{*}{} & \multicolumn{2}{|c|}{ Pre intervention } \\
\cline { 2 - 3 } & Range & Mean (SD) \\
\hline Overall responsiveness & $9-44$ & $23.56(13.46)$ \\
\hline
\end{tabular}

Table 03: Pre- interventional level of responsiveness for domains at medical clinic according to perception of patients

\begin{tabular}{|l|l|l|}
\hline $\begin{array}{l}\text { Domains of } \\
\text { Responsiveness }\end{array}$ & \multicolumn{2}{|c|}{ Pre intervention } \\
& Range & $4.72(2.67)$ \\
\hline Dignity & $2-8$ & $6.72(3.69)$ \\
\hline Communication & $3-12$ & $4.55(2.39)$ \\
\hline Autonomy & $2-8$ & $4.36(3.18)$ \\
\hline Confidentiality & $2-8$ & $0.92(1.68)$ \\
\hline Prompt Attention & $0-4$ & $2.29(1.25)$ \\
\hline Choice of provider & $0-4$ & $11.61(5.50)$ \\
\hline Basic Amenities & $6-24$ & \\
\hline
\end{tabular}

Table 04: Post- interventional level of overall responsiveness at medical clinic according to perception of patients

\begin{tabular}{|l|l|l|}
\hline & \multicolumn{2}{|c|}{ Post- intervention } \\
\hline & Range & Mean (SD) \\
\hline Overall responsiveness & $14-44$ & $32.05(6.29)$ \\
\hline
\end{tabular}


Table 05: Post- interventional level of responsiveness for domains at medical clinic according to perception of patients

\begin{tabular}{|l|l|l|}
\hline \multirow{2}{*}{ Domain } & \multicolumn{2}{|c|}{ Post intervention } \\
\cline { 2 - 3 } & Range & Mean (SD) \\
\hline Dignity & $3-8$ & $6.89(1.55)$ \\
\hline Communication & $5-12$ & $9.64(2.25)$ \\
\hline Autonomy & $2-8$ & $4.78(1.79)$ \\
\hline Confidentiality & $1-8$ & $6.87(1.63)$ \\
\hline Prompt Attention & $0-5$ & $1.55(1.95)$ \\
\hline Choice of provider & $1-4$ & $2.32(1.12)$ \\
\hline Basic Amenities & $6-24$ & $12.14(5.15)$ \\
\hline
\end{tabular}

Table 06: Impact of intervention on overall responsiveness

\begin{tabular}{|l|c|l|l|}
\hline & $\begin{array}{l}\text { Pre intervention } \\
\text { Mean }\end{array}$ & $\begin{array}{l}\text { Post intervention } \\
\text { Mean }\end{array}$ & $\begin{array}{l}\text { Significance } \\
\text { T test, P value }\end{array}$ \\
\hline Overall Responsiveness & 23.56 & 32.05 & $-11.193,0.001$ \\
\hline
\end{tabular}

Table 07: Impact of intervention on each domain of responsiveness

\begin{tabular}{|l|l|l|l|}
\hline Domain & $\begin{array}{l}\text { Pre- intervention } \\
\text { Mean(SD) }\end{array}$ & $\begin{array}{l}\text { Post - intervention } \\
\text { Mean(SD) }\end{array}$ & $\begin{array}{l}\text { Significance } \\
\text { T test, P value }\end{array}$ \\
\hline Dignity & $4.72(2.67)$ & $6.89(1.55)$ & $-13.74(0.000)$ \\
\hline Communication & $6.72(3.69)$ & $9.64(2.25)$ & $-13.21(0.000)$ \\
\hline Autonomy & $4.55(2.39)$ & $4.78(1.79)$ & $-1.516(0.130)$ \\
\hline Confidentiality & $4.36(3.18)$ & $6.87(1.63)$ & $-13.73(0.000)$ \\
\hline Prompt Attention & $0.92(1.68)$ & $1.55(1.95)$ & $-272 \quad(0.786)$ \\
\hline Choice of provider & $2.29(1.25)$ & $2.32(1.12)$ & $-1.36(0.174)$ \\
\hline Basic Amenities & $11.61(5.50)$ & $12.14(5.15)$ & $(0.000)$ \\
\hline
\end{tabular}


Table 08: Impact of intervention on patient waiting time in each step in minutes

\begin{tabular}{|l|l|l|}
\hline & $\begin{array}{l}\text { Pre test } \\
\text { Mean (SD) }\end{array}$ & $\begin{array}{l}\text { Post test } \\
\text { Mean (SD) }\end{array}$ \\
\hline $\begin{array}{l}\text { Waiting for } \\
\text { registration }\end{array}$ & $56.66(25.02)$ & $37.5(9.9)$ \\
\hline $\begin{array}{l}\text { Waiting before } \\
\text { consultation }\end{array}$ & $17.5(6.93)$ & $13(4.36)$ \\
\hline $\begin{array}{l}\text { Waiting in } \\
\text { consultation room }\end{array}$ & $28.3(13.5)$ & $17.8(5.83)$ \\
\hline $\begin{array}{l}\text { Obtaining next } \\
\text { appointment }\end{array}$ & $9.1(4.52)$ & $6.3(1.38)$ \\
\hline Waiting at pharmacy & $29.1(6-78)$ & $12(2.01)$ \\
\hline $\begin{array}{l}\text { Total waiting time } \\
\text { Significance } \\
\text { T test = 12.99, } \mathrm{p}=0.00\end{array}$ & $86.66(10.66)$ \\
\hline
\end{tabular}

\section{Annexure 01- Major delays identified in process mapping and causes for them}

\section{Delay 01 - There was a huge delay at patient registration.}

* One main reason for this delay was appointment system was being not functioned properly. Patients were given only date and time block (Exa; 8.00am-9.00am) during previous visit. They were not given their appointment number during previous visit. Therefore, all the patients belong to that one hour period gathered inside the clinic and huge congestion also noticed inside the clinic.

* Only one health care assistant was allocated to register the patients and registration table was placed inside the clinic premises.

Delay 02 - Another delay was for consultation

* Only doctors of one medical unit (4) were available to see the patients, there was big delay for consultation.

* Blood pressures of the patients were measured by using two manual pressure meters.

* As Patients were entering and exit to the consultation room by using the single door and there was a no unidirectional flow of patients. As a result of this, there was a huge congestion inside the clinic room and it causes further delay.

Delay 03 - Third delay was at Pharmacy

* Only two counters were open as pharmacists were not adequate.

* Delays in opening the pharmacy counters 


\section{Authors}

First Author - Ranga Sabhapathige, MBBS,MSc, MD,DIPPCA,,CHSS,University of Kent,UK, rangasabhapathige@ gmail.com. Second Author - D.A.R.K.Dilrukshi, BDS, MSc, MD, HNDIPPCA,qualifications, CHSS, University of

Kent,UK,deerasinghekanchana@gmail.com.

Correspondence Author - Ranga Sabhapathige, rangasabhapathige@ gmail.com, udeshmanoj@yahoo.com.,+44 07862740636. 